\title{
Etude de la structure de l'Emmental au cours de la fabrication, par la technique de microscopie électronique à balayage
}

\author{
M Rousseau, C Le Gallo \\ INRA, station de recherches laitières, 78350 Jouy-en-Josas, France
}

(Reçu le 29 juillet 1989; accepté le 9 novembre 1989)

\begin{abstract}
Résumé - L'observation au microscope électronique à balayage du fromage d'Emmental pendant l'égouttage et l'affinage montre que la caséine se présente dans le coagulum sous forme de micelles individualisées, puis se transforme, à la sortie du pressage, en une masse compacte qui évoluera ensuite en raison de la protéolyse et des modifications physico-chimiques de la pâte. Les globules gras fortement fragilisés par le chauffage et le brassage du caillé, perdent leur forme initiale au cours de l'affinage et se regroupent en amas volumineux aux formes diverses. Quelques joints de grain ont été observés, ainsi que la formation de microbulles de gaz, dont certaines peuvent être à l'origine de l'ouverture du fromage. L'observation de la flore interne est uniquement qualitative et ne donne qu'un faible aperçu de sa localisation au sein de la pâte en microcolonies nettement distantes les unes des autres.
\end{abstract}

\section{Emmental / égouttage / affinage / microstructure / MEB / cryo-MEB}

Summary - Scanning electron microscopic study of the structure of Emmental cheese during manufacture. A scanning electron microscopic study of Emmental cheese during draining and ripening stages has shown changes in casein structure. Initially the casein is found as individual micelles which are transformed into a compact mass which then undergoes further changes following proteolysis and other physico chemical modifications of the curd. Fat globules, which have become very fragile as a result of heat and agitation of the curd, also lose all their initial structure during ripening to give large masses with diverse forms. A few curd granules junctions have been observed as well as gas microbubbles which may be responsible for the "eye" formation of the cheese. Internal cheese flora in the curd was also observed qualitatively and microcolonies clearly well separated from each other were found.

\section{Emmental cheese / draining / ripening / microstructure / MEB / cryo-MEB}

\section{INTRODUCTION}

L'étude de la structure des trois grands types de fromage par la microscopie à balayage (MEB) est susceptible d'être inté- ressante à plusieurs titres. Elle donne, en premier lieu, une image de référence des structures normales du fromage étudié. Dans un $2^{e}$ temps, elle pourra aider à déterminer la cause de certains défauts présentés par ces fromages. 
Après avoir étudié l'évolution de la structure du Saint-Paulin au cours de la fabrication (Rousseau, 1988) nous examinons, ici, celle de l'Emmental. La technologie de ce fromage (Mocquot, 1979) est caractérisée par un chauffage du caillé qui permet d'obtenir un égouttage poussé et par suite une texture différente de celle des pâtes pressées. Les auteurs Rüegg et Blanc avaient fait en 1972 une étude de la structure de ce même fromage par deux techniques utilisées en parallèle : technique des coupes ultrafines et de cryofracture puis en 1979 (Blanc et al) et en 1980 par la technique de MEB. Cette dernière étude portait uniquement sur la période d'affinage et sur une pâte volontairement délipidée. L'évolution importante des techniques de préparation des échantillons et d'observation en MEB au cours de ces dernières années nous a incitées à la reprendre en incluant l'aspect du caillé pendant l'égouttage et en faisant une tentative d'observation de la matière grasse par l'utilisation d'échantillons cryofixés observés avec un MEB équipé d'un dispositif de refroidissement du porte-objet (cryo-MEB).

\section{MATÉRIEL ET MÉTHODES}

\section{Le fromage}

Les échantillons examinés provenaient de fromages d'Emmental fabriqués dans un atelier de grande capacité industrielle. Le lait standardisé, était thermisé à $63^{\circ} \mathrm{C}$ pendant $20 \mathrm{~s}$. La coagulation était obtenue par addition de présure au lait préalablement inoculé avec des levains de bactéries lactiques thermophiles et mésophiles. La coagulation et le travail en cuve étaient réalisés selon un mode habituel de fabrication. Les grains de caillé étaient chauffés jusqu'à $53^{\circ} \mathrm{C}$. Le moulage était pratiqué sous vide et sous sérum, et le temps de pressage était de $4 \mathrm{~h}$. Au démoulage le $\mathrm{pH}$ se situait entre 5,10 et 5,20 . Le salage était effectué pendant 24 h en sau- mure saturée, à $13^{\circ} \mathrm{C}$. L'affinage était effectué en 2 mois : 1 mois en cave froide (température de 10 à $12{ }^{\circ} \mathrm{C}$ ) avec brossage de la surface, toutes les 2 semaines, puis 3 à 4 semaines en cave chaude (température de $21^{\circ} \mathrm{C}$ ) jusqu'à l'obtention d'une ouverture correcte du fromage. II était ensuite conservé à la température de 4 à $5^{\circ} \mathrm{C}$ pendant 2 semaines.

\section{Préparation des échantillons pour la mi- croscopie}

\section{Echantillons déshydratés}

Pour la préparation des échantillons déshydratés, les prélèvements étaient pratiqués de la façon suivante pendant le travail en cuve : dans le coagulum, après décaillage et avant chauffage, pendant brassage et chauffage à $53^{\circ} \mathrm{C}$ en $30 \mathrm{~min}$, après brassage après le feu ( $45 \mathrm{~min})$, à la sortie du pressage, après salage; et pendant l'affinage : après 2 et 4 semaines en cave froide et $1,2,3$ et 4 semaines en cave chaude et 2 semaines en cave de conservation.

Pendant l'égouttage les prélèvements étaient pris à la périphérie et au centre du caillé et, pendant l'affinage, dans le tiers périphérique et au centre de la meule. Ils étaient immédiatement placés dans le 1 e fixateur (glutaraldéhyde à $2,5 \%$ tamponné à $\mathrm{pH} 7,2$ ) et envoyés au laboratoire en flacons fermés hermétiquement. A partir de ces morceaux de caillé ou de fromage, des parallélépipèdes de $1 \times 1 \times 3 \mathrm{~mm}$ étaient découpés et placés dans une nouvelle solution de glutaraldéhyde puis dans du tétroxyde d'osmium à $1 \%$. La déshydratation était faite dans l'éthanol et le séchage final par la méthode du point critique en $\mathrm{CO}_{2}$. Les échantillons déshydratés étaient fracturés et collés sur les porte-objets. Les surfaces fracturées étaient métallisées à l'or-palladium. L'observation était effectuée avec un microscope Hitachi S-450, sous une tension de 15 ou $20 \mathrm{kV}$. Le détail de ces techniques est décrit par Cohen (1974) et par Rousseau (1988).

\section{Echantillons cryofixés}

Les échantillons soumis à une congélation étaient prélevés après le salage, après un sé- 
jour de 15 jours en cave froide et après 30 jours en cave chaude. Ils étaient maintenus entre $4^{\circ} \mathrm{C}$ et $10^{\circ} \mathrm{C}$ pendant le transport et jusqu'à l'observation au microscope (environ $48 \mathrm{~h}$ ). Aucune fixation chimique n'était pratiquée. Des baguettes de fromage de $1 \times 1 \times 6 \mathrm{~mm}$ étaient placées verticalement dans les logements d'un porte-objet et collées. La cryofixation était effectuée dans l'azote pâteux à $-210^{\circ} \mathrm{C}$. La fracture et la métallisation des surfaces fracturées avaient lieu dans la chambre d'un appareil Emscope SP 2000A où une température de $-190^{\circ} \mathrm{C}$ était maintenue grâce à une circulation d'azote liquide et à la présence d'un vide de l'ordre de $10^{-1} \mathrm{~mm} \mathrm{Hg}$. Un dispositif de transfert, parfaitement étanche, permettait de maintenir le même vide et la même température que dans la chambre de fracture pour transporter l'échantillon de la chambre de préparation jusqu'au porte-objet du microscope, qui était refroidi à - $160^{\circ} \mathrm{C}$ par une circulation d'azote liquide. L'observation était faite sous une tension de 15 kV avec un microscope à balayage JEOL, JSM$840 \mathrm{~A}$. Nous présentons ici, uniquement des échantillons congelés, non sublimés : les essais de sublimation effectués sur l'Emmental ne donnaient pas de résultats reproductibles.

\section{RÉSULTATS}

Pour l'étude de la caséine, nous utilisions des échantillons déshydratés, prélevés pendant la période d'égouttage et pendant l'affinage. Pour l'étude des globules gras, l'observation d'échantillons cryofixés était plus appropriée, évitant l'utilisation de solvants, susceptibles de solubiliser la matière grasse. Dans ce dernier cas, seuls des échantillons prélevés pendant l'affinage étaient préparés et examinés.

\section{Aspect de la caséine}

Sur les échantillons déshydratés, la structure de la caséine présentait un changement important pendant toute la période d'égouttage c'est-à-dire jusqu'à la fin du pressage. D'un réseau lâche de fines particules dont le diamètre moyen est de $100 \mathrm{~nm}$, on passait, après le premier brassage avant chauffage, à un réseau un peu plus serré, mais formé de particules semblables aux précédentes (fig 1). Après chauffage à $53^{\circ} \mathrm{C}$, les petites particules de $100 \mathrm{~nm}$ n'étaient plus apparentes, car elles s'étaient soudées en paquets d'environ $700 \mathrm{~nm}$ de diamètre, assemblés le plus souvent en chaînes (fig 2). L'échantillon prélevé à la fin du travail en cuve montrait un aspect comparable à celui du précédent quoique la structure de la caséine soit en partie masquée par la matière grasse en cours d'extraction par les solvants (fig 3). A la fin du pressage, la structure en chaînes avait disparu et la caséine formait alors une masse compacte dans laquelle apparaissaient les cavités où étaient initialement logés les globules gras, détruits par les solvants de la déshydratation (fig 4). L'aspect du caillé après salage était le même qu'à la sortie du pressage. Cette structure de la caséine, compacte et lisse, ne se modifiait pas pendant tout le séjour en cave froide (fig 5) et la 1 e moitié du séjour en cave chaude. C'était au cours de la $2^{\mathrm{e}}$ quinzaine en cave chaude que la structure de la caséine devenait progressivement grumeleuse (figs 6 et 7 ). Elle se maintenait ensuite dans cet état pendant le séjour en cave de conservation à basse température.

Sur les échantillons observés après congélation sans sublimation, la phase liquide solidifiée et la caséine ne se différenciaient pas l'une de l'autre. De tels échantillons ne pouvaient donc apporter aucun renseignement sur la structure de la caséine. En effet, sur les figs 8 et 9 , la pâte qui enrobe les globules gras présente une structure homogène du début à la fin de l'affinage. 

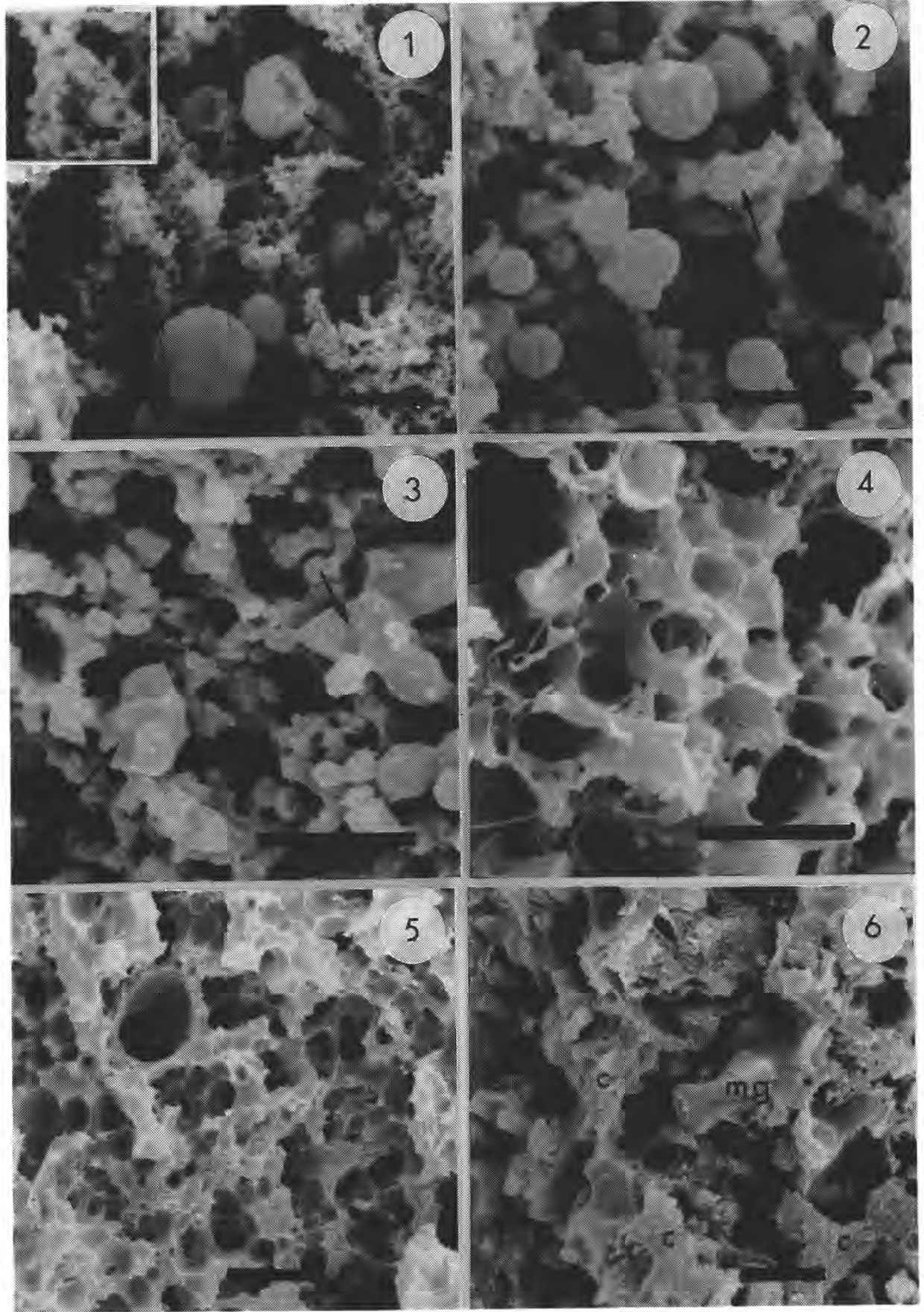


\section{Aspect des globules gras}

Dans les premiers échantillons déshydratés, malgré l'utilisation de solvant, les globules gras étaient assez nombreux (figs 1 et 2). Ils apparaissaient sphériques, bien isolés les uns des autres, avec une surface lisse et certains présentaient des dépressions plus ou moins importantes (fig 1 , flèches). Dans l'échantillon prélevé après chauffage, les globules gras étaient beaucoup plus modifiés par les solvants et apparaissaient vides ou bien accolés en paquets (fig 3). Dans le prélèvement effectué en fin de pressage, l'extraction de la matière grasse était totale, et il ne subsistait que des cavités vides dans la masse compacte de la caséine (fig 4). Cet aspect de la pâte pouvait encore être observé pendant la période d'affinage depuis le salage jusqu'à la fin de la $2^{\theta}$ semaine de cave chaude. C'est en effet au cour de la $2^{\ominus}$ moitié du séjour en cave chaude que l'aspect de la pâte changeait. Les cavités des globules gras étaient très déformées et la matière grasse, qui avait totalement perdu son aspect globulaire, était incomplètement extraite (figs 6 et 7 ).

Fig. 1. Coagulum découpé et brassé avant chauffage. La structure est demeurée la même que celle du coagulum initial mais la caséine est légèrement plus resserrée. Les globules gras ont le même aspect que dans le coagulum initial, certains présentent des altérations (flèches). Barre $=5 \mu \mathrm{m}$. Encart : détail caséine. Barre $=0,5 \mu \mathrm{m}$.

Milk curd after cutting and stirring just before heating. Structure is close to that of initial curd but casein is slightly more compact. Milk fat globules have the same aspect as in the initial coagulum, but some globules show alterations (arrow). Bar $=5 \mu \mathrm{m}$. Window: casein detail, bar $=0.5 \mu \mathrm{m}$.

Fig. 2. Grain de caillé au cours du chaufiage. Les micelles de caséine, peu apparentes, sont regroupées en paquets (flèches), eux-mêmes assemblés en chaînes. Noter la présence de nombreux globules gras non encore extraits. Barre $=5 \mu \mathrm{m}$.

Curd grain during heating. Casein micelles are not very individualized and are grouped together in clusters which are arranged in chains (arrows). Note the presence of numerous milk fat globules which have not yet been extracted. Bar $=5 \mu \mathrm{m}$.

Fig. 3. Grain de caillé après chauffage. La caséine a le même aspect que sur la fig 2 mais est légèrement enrobée et masquée par la matière grasse en cours d'extraction. Noter la déformation et l'accolement des globules par l'action des solvants (flèches). Barre $=5 \mu \mathrm{m}$.

Curd grain after heat treatment. Casein has the same aspect as in fig 2 but is slightly coated and partially masked by the milk fat in the process of being extracted. Note the deformation and the clustering of fat globules due to the action of solvents (arrows). Bar $=5 \mu \mathrm{m}$.

Fig. 4. Fromage au sortir de la presse, avant salage. Observer l'aspect compact de la caséine et l'absence totale de globules gras, leurs logements témoignent seuls de leur présence. Barre $=5 \mu \mathrm{m}$.

Cheese just after pressing but before salting. Note the casein compaction and total absence of milk fat globules; only the cavities formerly occupied by fat are observed. Bar $=5 \mu \mathrm{m}$.

Fig. 5. Fromage en cours d'affinage, après 2 semaines en cave froide. L'aspect général de la pâte est peu différent de celui observé à la sortie du pressage et subsistera jusqu'à la $2 e$ semaine de cave chaude. Barre $=5 \mu \mathrm{m}$.

Cheese during ripening, after 2 weeks in a cold ripening room. The general aspect of the paste is slightly different from that observed at the end of pressing; this subsists until the end of the 2nd week in warm ripening room. Bar $=5 \mu \mathrm{m}$.

Fig. 6. Fromage en cours d'affinage après 4 semaines en cave chaude. Noter la différence de structure entre la pâte du fromage à ce stade et celle que présentait le fromage en cave froide. Les globules gras sont souvent rassemblés en masses importantes incomplétement extraites par les solvants (m.g.) et la caséine a perdu sa structure lisse (c.). Barre $=5 \mu \mathrm{m}$.

Cheese during ripening, after 4 weeks in a warm ripening room. Note the difference in structure between cheese paste at this stage and that observed in cheese in a cold ripening room. The milk fat globules are often assembled in large clusters which have not been completely extracted by solvents (mg). Casein has lost its smooth structure (c). Bar $=5 \mu \mathrm{m}$. 

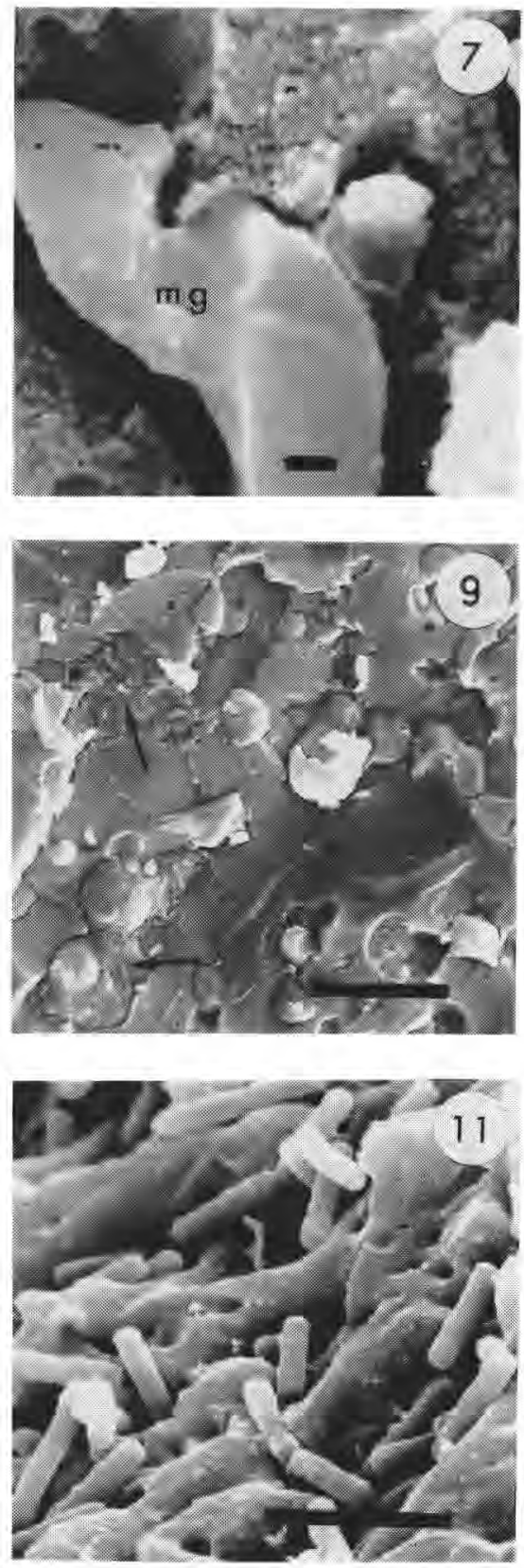
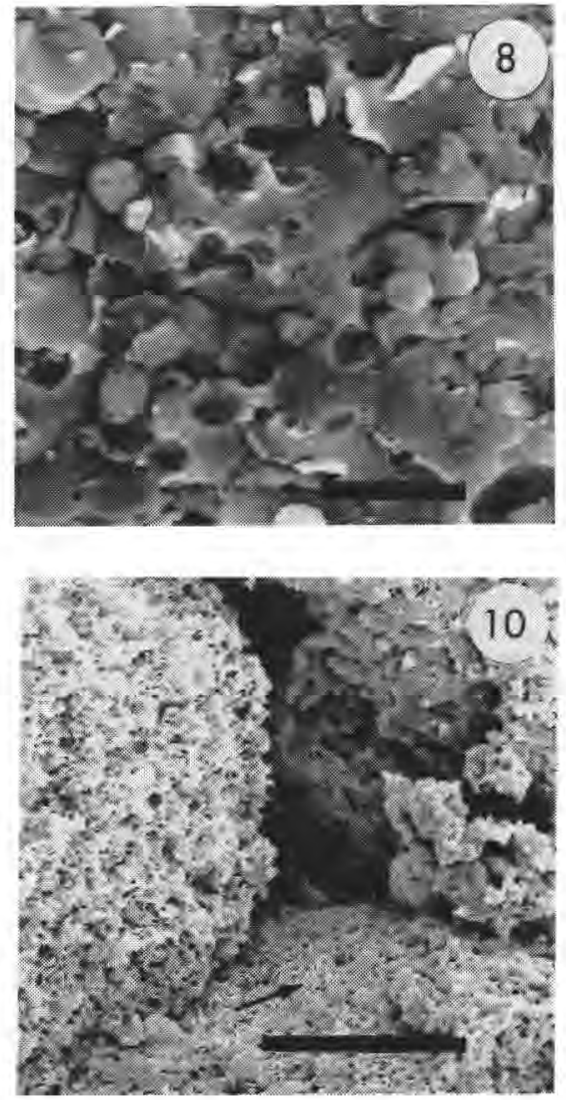

Fig. 7. Fromage en cours d'affinage après 4 semaines en cave chaude. Détails de structure. Matière grasse non extraite (m.g.) et caséine grumeleuse (c.). Barre $=0,5 \mu \mathrm{m}$.

Cheese during ripening after 4 weeks in warm ripening room. Structural details. Non extracted milk fat (mg) and crumbly casein (c). Bar $=0.5$ $\mu \mathrm{m}$.

Fig. 8. Surface de fracture d'un échantillon congelé. Fromage à la sortie du salage. Les globules gras sont souvent assemblés en groupes de 3 ou 4 mais restent bien individualisés. Barre $=10 \mu \mathrm{m}$.

Fracture surface of a deep frozen sample just after salting. Milk fat is often assembled in groups of 3 or 4 globules which remain well individualized. $\mathrm{Bar}=10 \mu \mathrm{m}$.

Fig. 9. Fracture d'un échantillon congelé. Fromage après un séjour de 4 semaines en cave chaude. Quelques globules restent encore isolés mais la plupart sont regroupés en amas (flèches). Barre = $10 \mu \mathrm{m}$.

Fracture of a deep frozen sample. Cheese after 4 weeks in warm ripening room. Some globules still remain isolated; but most are grouped in clusters (arrows). Bar $=10 \mu \mathrm{m}$.

Fig. 10. Joints de grain dans un fromage à la sortie du pressage. Aspect d'ensemble. Zone riche en bactéries (flèches). Barre $=50 \mu \mathrm{m}$.

Space between curd particles just after pressing. General aspect. Numerous bacteria are present (arrows). Bar $=50 \mu \mathrm{m}$.

Fig. 11. Détail de la figure précédente montrant la zone riche en bactéries. Barre $=5 \mu \mathrm{m}$.

Detail of the previous figure showing the area with a high level of bacteria (arrow). Bar $=5 \mu \mathrm{m}$. 
Ces observations faites après déshydratation des échantillons, étaient les mêmes dans les échantillons prélevés au centre et à la périphérie de la meule.

Sur les échantillons préparés par cryofixation et observés congelés, les globules gras apparaissaient bien présents sur les surfaces de fracture des échantillons d'affinage, contrairement à ce qui était observé sur les échantillons déshydratés. Les formes sphériques des globules étaient, là encore, plus nombreuses au début de l'affinage (fig 8), qu'à la fin (fig 9). Dans ces échantillons de fin d'affinage la matière grasse était rassemblée en paquets volumineux qui résultaient de l'accolement de plusieurs globules. Au niveau de ces amas, le plan de fracture passait plus souvent dans la masse, qu'à la surface (fig 9, flèches).

\section{Observation des joints de grain}

Quelques joints de grain étaient observés dans le caillé, à la sortie du pressage sur les échantillons déshydratés (fig 10). La structure du caillé délimitant les joints était la même que celle du caillé à l'intérieur des grains : la moitié gauche de la fig 10 présente la même structure, à plus faible grandissement, que celle des figs 4 et 5 . La partie inférieure du grain, sur la fig 10 , et la paroi de droite présentent une structure différente de la caséine dû au fait que celle-ci était recouverte par de la matière grasse incomplètement extraite. La fig 11 représente la base du joint riche en bactéries ayant la morphologie des lactobacilles. L'autre joint observé (fig non représentée) était dépourvu de germes. Des images de soudures de grains n'étaient pas observées sur les échantillons déshydratés de fin d'affinage.

\section{Formation des bulles de gaz}

A la fin de la dernière semaine du séjour en cave froide, commençaient à apparaître dans la pâte, des cavités sphériques de diamètres variant de 30 à $50 \mu \mathrm{m}$ (figs 12 et 13). Leur nombre augmentait pendant les premières semaines de cave chaude, puis diminuait progressivement.

\section{Observations des micro-organismes dans le fromage pendant l'affinage}

Les micro-organismes observés en début d'affinage étaient des streptocoques (fig. 14) regroupés en microcolonies de 10 à $15 \mu \mathrm{m}$ de diamètre séparées en moyenne par 150 à $200 \mu \mathrm{m}$. Les lactobacilles (fig 15), plus rares, se regroupaient en colonies distantes les unes des autres de 500 à $600 \mu \mathrm{m}$. La quantité de germes par colonie, pour l'une ou l'autre espèce, était difficilement appréciable, elle pouvait varier de 50 jusqu'à 500 germes. Au cours du séjour en cave froide, ces germes devenaient plus rares, en colonies souvent mixtes, et présentaient des formes peu altérées. En fin de séjour en cave chaude des formes coccoïdes très altérées étaient observées (fig 16) et des formes du type lactobacilles, peu altérées, étaient très rarement observées. Les bactéries propioniques n'étaient jamais observées sur les échantillons, leur nombre étant sans doute inférieur au seuil de détection des bactéries dans une pâte de fromage observée au MEB, seuil qui se situe à environ $1.10^{9}$ bactéries par $\mathrm{g}$ de fromage (Rousseau, 1984). 

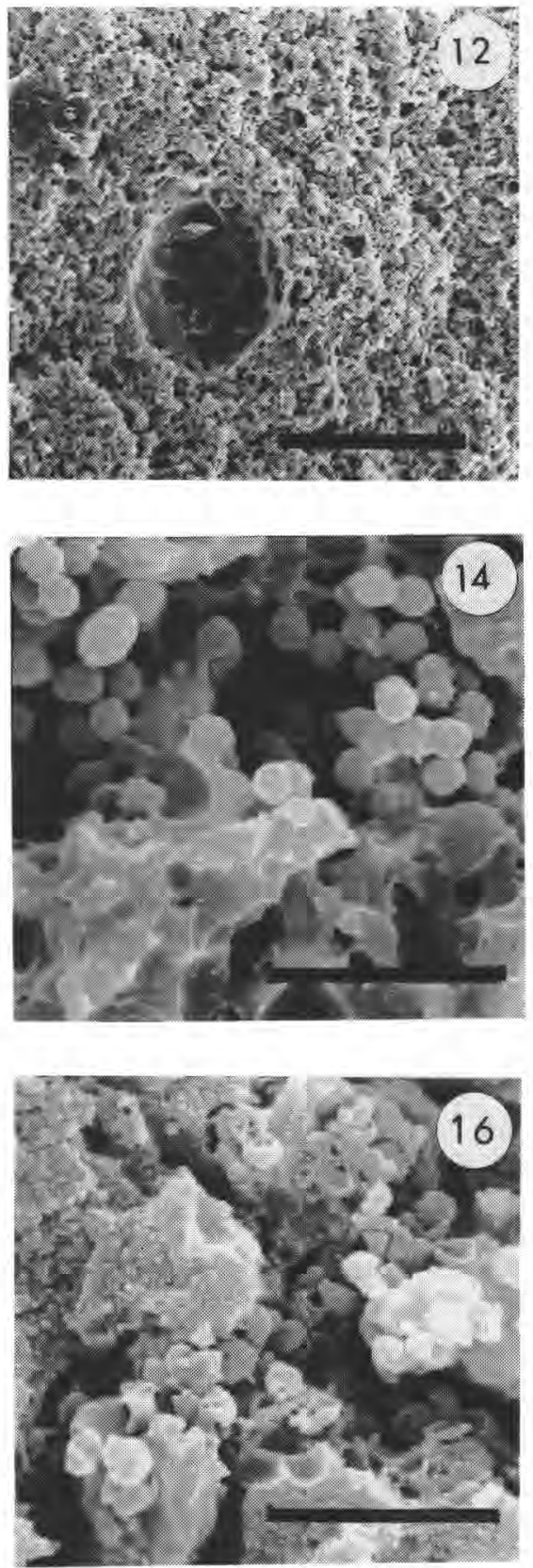
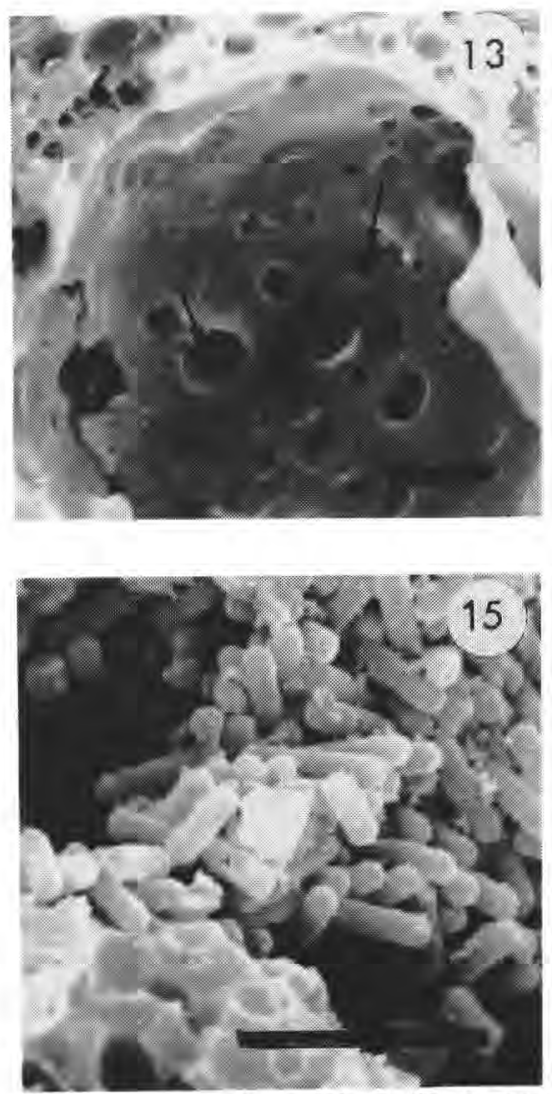

Fig. 12. Morphologie d'une microbulle de gaz dans un fromage ayant passé 8 jours en cave chaude. Barre $=50 \mu \mathrm{m}$.

Morphology of a gas microbubble in a cheese which has remained for 8 days in a warm ripening room. Bar $=50 \mu \mathrm{m}$.

Fig. 13. Détail d'une microbulle: noter, sur sa paroi, les cavités antérieurement occupées par les globules gras (flèches). Barre $=5 \mu \mathrm{m}$.

Detail of a microbule. On the wall, note the cavities previously occupied by milk fat globules (arrows). Bar $=5 \mu \mathrm{m}$.

Fig. 14. Microcolonie de streptocoques lactiques thermophiles dans le caillé prélevé à la fin du pressage. Barre $=5 \mu \mathrm{m}$.

Microcolony of thermophilic lactic streptococci in the curd examined at the end of pressing. Bar = $5 \mu \mathrm{m}$.

Fig. 15. Microcolonie de lactobacilles thermophiles, observée dans un fromage ayant séjourné 15 jours en cave froide. Barre $=5 \mu \mathrm{m}$.

Microcolony of thermophilic lactobacilli in a cheese which has remained for 15 days in a cold ripening room. Bar $=5 \mu \mathrm{m}$.

Fig. 16. Aspect d'une colonie de cocci très altérés dans un fromage ayant séjourné 3 semaines en cave chaude. Barre $=5 \mu \mathrm{m}$.

Aspect of a markedly altered cocci colony in a cheese which has remained for 3 weeks in a warm ripening room. Bar $=5 \mu \mathrm{m}$. 


\section{DISCUSSION}

\section{Caséine}

La technique des échantillons déshydratés nous permet d'observer une évolution rapide de la structure de la caséine au cours de l'égouttage, les modifications étant ensuite plus lentes pendant l'affinage.

La préservation de la structure de la caséine dans les échantillons déshydratés est due à la fixation en glutaraldéhyde, bien connue pour son rôle de protection vis-à-vis des structures protéiques (Hayat, 1981; Cohen, 1974). L'aspect des micelles, observé en début d'égouttage, correspond aux descriptions précédemment publiées par différents auteurs (Kalab et Harwalkar, 1973; Glaser et al, 1980; Ohashi et al, 1983). En effet la taille des particules décrites sur les figs 1 et 2 correspond à la taille des micelles de caséine évaluée par différentes techniques, taille comprise entre 0,1 et $0,6 \mu \mathrm{m}$ (Holt, 1985).

Cette structure micellaire disparaît au cours de la cuisson du caillé. Le pressage transforme la caséine en une masse compacte creusée par les logements des globules gras et par les canaux de circulation du lactosérum. Dans cette masse protéique fracturée on n'observe aucune trace des limites des micelles, car celles-ci sont agglomérées et leur fusion semble totale. Ceci est en accord avec l'aspect des structures de caséine d'un fromage jeune, obtenues sur coupes, au microscope électronique à transmission par Rüegg et Blanc (1972) et nous-mêmes (résultats non publiés).

L'aspect grumeleux de la caséine à la fin de l'affinage, nettement visible sur la fig 7, révèle une désagrégation de la masse protéique en particules d'environ 50 à $100 \mathrm{~nm}$ de diamètre. Les particules pa- raissent un peu plus volumineuses que les submicelles. Ce nouvel aspect suppose un réarrangement de la phase aqueuse et de la caséine pendant la période d'affinage. Ce réarrangement est vraisemblablement une conséquence de la protéolyse. Des petits peptides et des acides aminés libres sont mis en évidence dans les extraits de fromage, à la fin de l'affinage (Steffen et al, 1987) : 22 à $25 \%$ de l'azote protéique total est transformé en azote soluble; une quantité importante de gros peptides est également détectée après filtration sur gel (Blanc et al, 1979). Cette dégradation des caséines entraîne des modifications physico-chimiques de la pâte, notamment une diminution de l'activité de l'eau et par suite une augmentation de son adsorption (Blanc et al, 1979). Cet état de la caséine la rend plus plastique et par suite plus apte à subir des déformations résultant de l'action d'une pression croissante due à l'accumulation de gaz carbonique produit au cours de la fermentation propionique. La comparaison de la fig 5 (caséine au début de l'affinage) et de la fig 6 (caséine en fin de séjour en cave chaude) montre bien l'importance de ces déformations.

L'observation de la caséine sur les échantillons cryofixés, non sublimés, nous l'avons déjà noté, n'apporte aucun renseignement sur l'évolution de sa structure.

\section{Matière grasse}

En ce qui concerne la matière grasse, les deux sortes d'échantillons (déshydratés et cryofixés) étaient préparés.

Dans le cas des échantillons déshydratés, l'absence de solubilisation de la matière grasse dans les échantillons prélevés au début de la fabrication, avant chauffage, est surprenante. Une constatation analogue avait été faite sur des fromages 
de Saint-Paulin observés antérieurement avec la même technique de préparation (Rousseau, 1988). Les solvants n'avaient en effet pratiquement pas d'action sur la matière grasse, dans les échantillons prélevés avant le pressage. Ces observations suggèrent que la perméabilité de la membrane des globules était fortement modifiée pendant la fabrication du caillé. De plus, cette membrane était certainement fragilisée avant la fabrication, par le refroidissement et le brassage du lait (Mulder et Walstra, 1974); l'aspect de certains globules, observés dans le caillé, comparables à des "ballons partiellement dégonglés", en témoigne (fig 1). Cela explique l'impossibilité d'effectuer une observation des globules gras jusqu'à la fin de l'affinage avec cette méthode de préparation des échantillons.

La technique de congélation rapide à très basse température des échantillons permet de figer les structures en l'état, au moment de la cryofixation (Robards et Sleytr, 1985). L'emploi de ce procédé, excluant tout traitement chimique, semblait plus favorable à l'observation des globules gras in situ, dans la pâte du fromage, pendant l'affinage. En effet, malgré la perte de certains globules par arrachement, au moment de la fracture de l'échantillon, on peut observer la variation de leur répartition entre le début et la fin de l'affinage. On constate une certaine similitude entre la forme des cavités sur les échantillons déshydratés à la fin de l'affinage et la forme des amas de matière grasse observés sur les échantillons cryofixés en fin d'affinage également. Le changement de forme de ces cavités est vraisemblablement dû à l'augmentation de la pression interne dans les fromages au cours de l'affinage. Cette pression provoque des mouvements dans la pâte, ces derniers favorisant la coalescence de plusieurs globules entre eux et leur déformation.

\section{Joints de grains de caillé}

L'observation des quelques joints de grain décrits dans les résultats ne révèle pas une structure particulière du caillé à leur niveau, ni une richesse en bactéries très importante. Ces joints observés étaient situés à l'intérieur du caillé. Des joints localisés près de la surface d'un fromage insuffisamment égoutté pourraient former des petites poches, au degré d'humidité élevé, favorables au développement de moisissures ou autres micro-organismes indésirables provenant de l'environnement du fromage.

\section{Microbulles de gaz}

La formation de cavités régulièrement sphériques dont le diamètre est supérieur à celui des globules gras, à la fin du séjour en cave froide et au début du passage en cave chaude, paraît correspondre à l'apparition de l'ouverture dans le fromage (Bergère et Accolas, 1986). En effet on ne trouve pas ces cavités dans les premiers échantillons prélevés après pressage et elles ne résultent donc pas de l'emprisonnement d'air au moment du soutirage. Ceci est normal puisque les fromages sont moulés sous vide et sous sérum. Ces cavités ne peuvent pas être confondues, non plus, avec les logements de la matière grasse résultant de la fusion de plusieurs globules gras en raison de leurs formes très différentes. II semble donc qu'elles soient dues à la production de gaz par les fermentations bactériennes. Des cavités semblables ont déjà été observées par Blanc et al (1979) dans l'Emmental. La période d'apparition de ces microbulles semble trop précoce pour attribuer leur formation uniquement au développement propionique. II pourrait s'agir d'une produc- 
tion ménagée de gaz carbonique au cours de la fermentation lactique sous presse et pendant les jours suivants ce qui confirmerait les observations de Flückiger et Walser (1977). L'augmentation du nombre de ces bulles pendant le séjour en cave chaude correspondrait alors à la fermentation propionique. Enfin leur raréfaction pendant les dernières semaines d'affinage suggère qu'une migration du gaz de ces cavités s'opère et aboutit à la formation de l'ouverture définitive. II est donc très probable que certaines de ces microbulles représentent les "germes" initiateur des "yeux» de l'Emmental affiné.

\section{Présence des bactéries}

L'observation au microscope électronique à balayage ne permet pas la détection des germes pour des valeurs inférieures à $1 x$ $10^{9}$ bactéries par $\mathrm{g}$ de caillé ou de fromage, ce qui explique la difficulté à observer les lactobacilles et l'absence totale, apparente, des bactéries propioniques. Les études faites par des méthodes bactériologiques (Mocquot, 1979) sont seules valables pour une estimation quantitative de la flore. Toutefois l'observation au microscope nous a apporté une image de la taille des microcolonies et de leur répartition dans la pâte de l'Emmental, et ces caractéristiques observées ici, sont les mêmes que celles qui sont rencontrées dans une pâte de Saint-Paulin en cours d'affinage. La dégradation de la caséine par ces bactéries reste très localisée au niveau des colonies (zones vides) et sa mise en évidence en MEB sur les échantillons déshydratés (figs 14-15-16) n'est pas très facile à cause des nombreuses cavités correspondant aux logements des globules gras existant dans la pâte. La destruction de la caséine par la flore interne du fromage, également mise en évidence sur coupes minces et par cryofracture par Rüegg et Blanc (1972), ne représente qu'une zone très restreinte au contact immédiat des cellules bactériennes. Une attaque partielle de la caséine dans l'ensemble de sa masse, responsable de l'aspect grumeleux observé vers la $3 e$ semaine de cave chaude, et en action simultanée avec les transformations physicochimiques dont nous avons déjà parlé, pourrait être un résultat de la diffusion lente et en faible quantité des enzymes protéolytiques de ces bactéries.

\section{REMERCIEMENTS}

Les auteurs remercient très vivement JP Accolas pour ses critiques et nombreuses suggestions. Leurs remerciements vont également à $M$ Desmazeaud et $L$ Vassal. Nous avons été sensibles à l'excellent accueil qui nous a été réservé par Mme D Guillaumin et le Pr P Cassier du Service de Microscopie Electronique du CNRS (105, Bd Raspail, Paris) pour l'utilisation du microscope à balayage à platine refroidie. Des remerciements sont également adressés à M. JR Kerjean (ITG-Ouest) qui a eu l'amabilité de nous procurer les échantillons d'Emmental.

\section{RÉFÉRENCES}

Bergère JL, Accolas JP (1986) Non-sporing and sporing anerobes in dairy products. In: $A n$ aerobic Bacteria in Habitats other than Man (Barnes EM, Mead GC, eds) Blackwell, Oxford, pp 373-382

Blanc B, Ruëgg M, Baer A, Casey M, Lukesch A (1979) Essais comparatifs dans le fromage d'Emmental avec et sans fermentation secondaire. Schweiz Milchwirtsch Forsch 8, 27 36

Cohen AL (1974) Critical point drying. The processing of specimens. In: Principles and Techniques of Scanning Electron Microscopy. Vol. 1. Biological Applications (Hayat MA, ed). Van Nostrand Reinhold, New York, pp 71-76 
Flückiger E, Walser F (1977) Beitragzur Kermtnis der $\mathrm{CO}_{2}$-bildung von Rohmicshkulturen. Schweiz Milchztg 103, 640-641

Glaser J, Carroad PA, Dunkley WL (1980) Electron microscopic studies of casein micelles and curd microstructure in cottage cheese. $J$ Dairy Sci 63, 37-48

Hayat MA (1981) Fixation for Electron Microscopy, Academic Press, New York, pp 77-87

Holt MA (1985) The size distribution of bovine casein micelles. A review. Food Microstruct 4, 1-10

Kalab M, Harwalkar VR (1973) Milk gel structure. I. Application of scanning electron microscopy to milk and other food gels. J. Dairy Sci. 56, 835-842

Mocquot G (1979) Swiss-type cheese. J Dairy Sci 46, 133-160

Mulder H, Walstra $P$ (1974) The fat globule membrane. In: The Milk Fat Globule (Mulder $\mathrm{H}$, Walstra $\mathrm{P}$, eds). Pudoc, Wageningen, The Netherlands, pp 88-93

Ohashi T, Haga S, Yamanchi K, Olson NF (1983) Scanning electron microscopy of casein micelle networks in milk rennet curd. Jpn J Zootech Sci 54, 479-481
Robards AW, Sleytr UB (1985) Direct viewing and analysis of cold specimens. In: Low Temperature Methods in Biological Electron Microscopy (AM Glauert, ed). Elsevier, Amsterdam, ch 3, pp 158-177

Rousseau M (1984) Study of the flora of traditional Camembert cheese by scanning electron microscopy. Milchwissenschaft 39, 129134

Rousseau M (1988) Changes in the microstructure of Saint-Paulin cheese during manufacture studied by scanning electron microscopy. Food Microstruct 7, 105-113

Rüegg M, Blanc B (1972) Beiträge zur elektronenmikroskopischen struktur der labgallerte und des Käseteiges. Schweiz Milchwirtsch Forsch 9, 1-8

Rüegg M, Moor U, Blanc B (1980) Veränderungen der Feinstruktur von Greyerzerkäse in verlauf der Reifung. Eine studie mit dem Rasterelectronmikroskop. Milchwissenschaft 35, 329-335

Steffen C, Flückiger E, Bosset JO, Rüegg M (1987) Swiss-type varieties. In: Cheese: Chemistry, Physics and Microbiology. Vol. 2 (Fox PF, ed). Elsevier, London, pp 93-120 